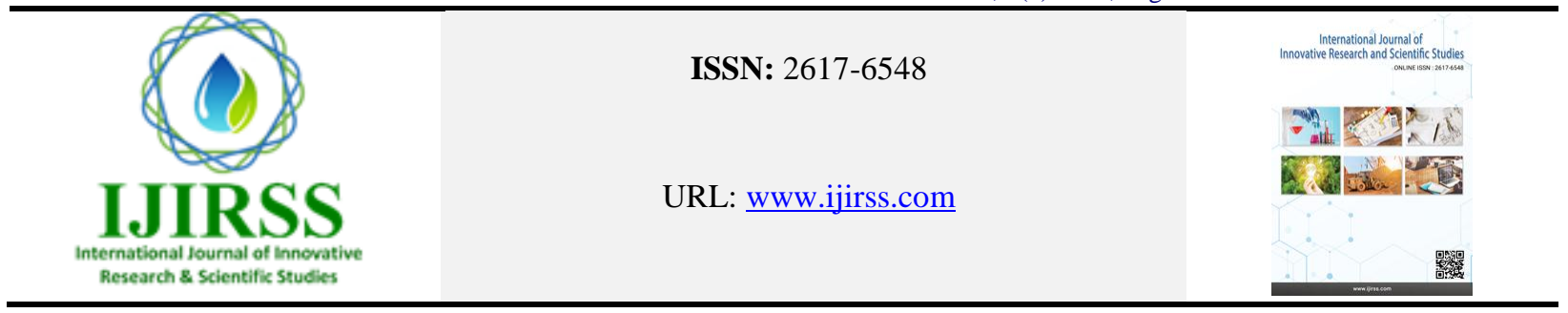

\title{
Investigation of Mavlana Aznab Sheberghani School Students' Reluctance in Math Subject
}

\author{
Muhammad Rafi Nazari \\ General Technical Subjects Department, Geology and Mines Faculty, Jawzjan University, Jawzjan, Afghanistan \\ (Email: rafi.nazary@yahoo.com)
}

\begin{abstract}
The subject of this study is to investigate causes of students' disinterest in education department or domain (secondary school of Mavalana Aznab Sheberghani). The essential and important objective of this study is to identify causes of students' disinterest in math subject. This study was a filed and applied research. The instrument was questionnaire and filled in by students in the field. One of the reasons of students' disinterest in math subject in secondary school is weaknesses in foundation and mathematical thinking. In math instruction, these are the problems that need to be considered: to have professional teachers, qualified and strong management and leadership based on the system of reward and punishment, use of technology in teaching math subject, and use of appropriate curriculum based on society's needs. These are the primary needs of our society. The importance of theoretical research is to examine various theories on the title of study (causes of students' disinterest in math subject) and find out different variables and answer to the research questions appropriately. The importance of research is to find out the causes of lack of progress in learning math subject. The results indicate that there are a lot of barriers and problems in math learning among students. It is important to increase students' interests and motivation by eliminating the problems.
\end{abstract}

Keywords: Disinterest, Professional, Instruction, lesson Plan, Math.

DOI: 10.53894 /ijirss.v4i3.72

Funding: This study received no specific financial support.

History: Received: 23 February 2021/Revised: 8 April 2021/Accepted: 5 May 2021/Published: 20 May 2021

Licensed: This work is licensed under a Creative Commons Attribution 4.0 License $(\mathrm{cc})$ EY

Competing Interests: The author declares that there are no conflicts of interests regarding the publication of this paper.

Transparency: The author confirms that the manuscript is an honest, accurate, and transparent account of the study was reported; that no vital features of the study have been omitted; and that any discrepancies from the study as planned have been explained.

Ethical: This study follows all ethical practices during writing. 


\title{
بررسى عو امل كم علاقه كى شاكردان دوره متوسطه مكتب مولانا اذنب شبرغانى به مضمون رياضى
}

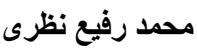 \\ ديبارتمنت مضامين عمومى تخنيكى، دانشكده جيولوجى ومعادن، دانشكاه جوزجان، جوزجان، افغانستان \\ خلاصه
}

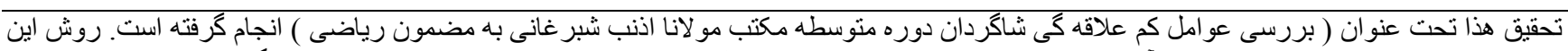

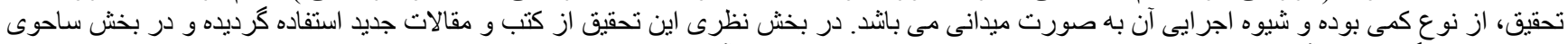

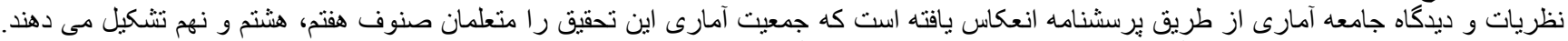

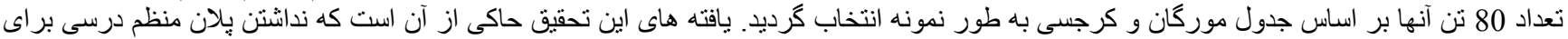

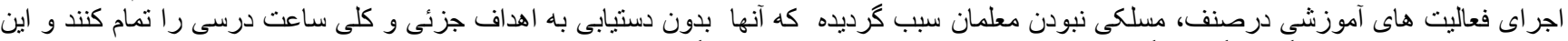

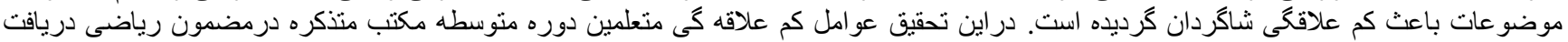

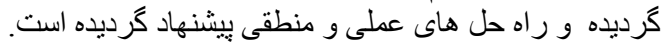

كلمات كليلى: كم علاقه كى، مسلكى، آموزش، بِلان درسى، رياضى

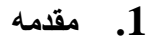

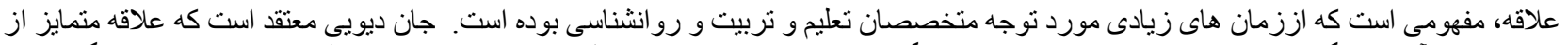

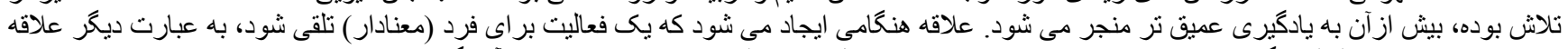

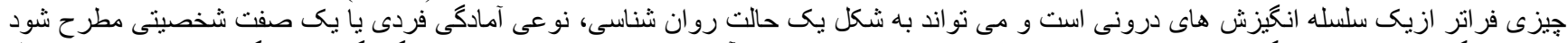

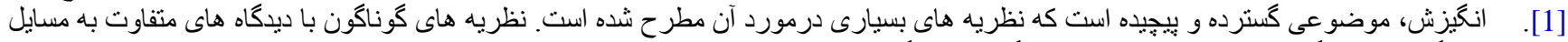

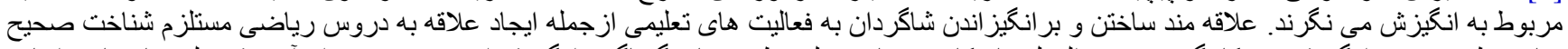

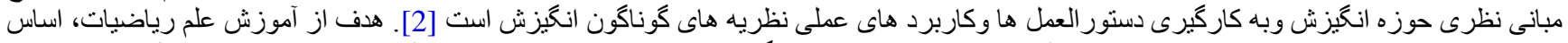

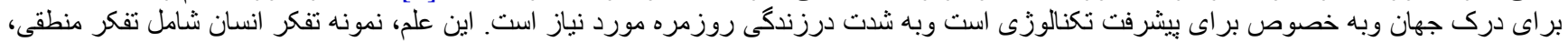

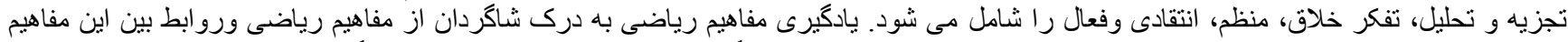

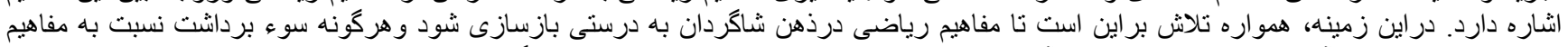

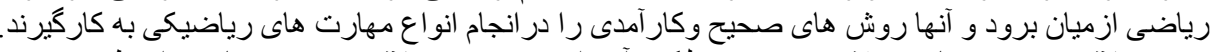

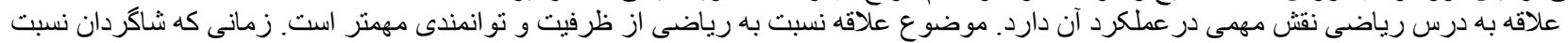

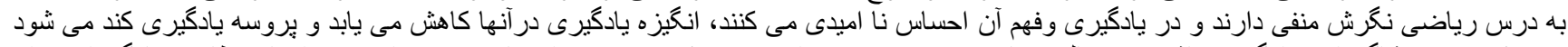

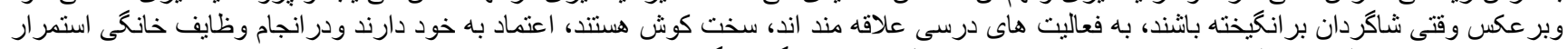

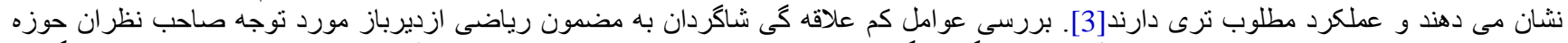

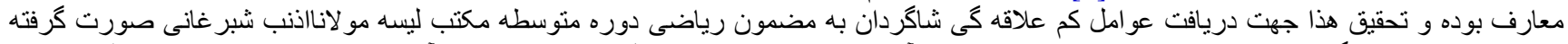

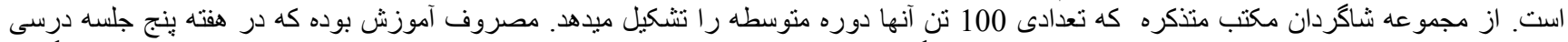

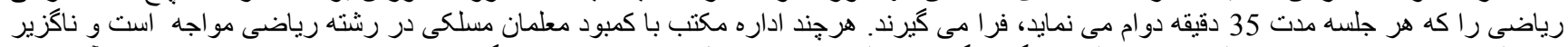

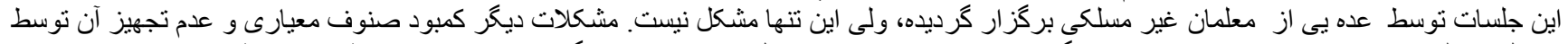

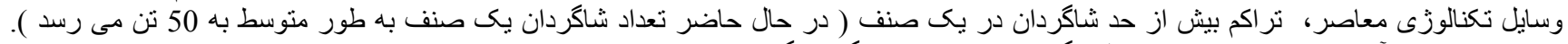

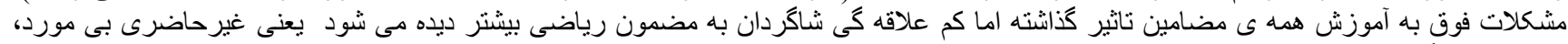

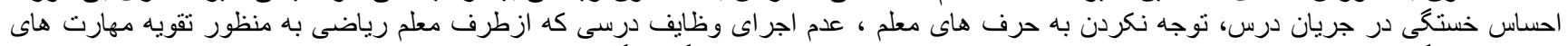

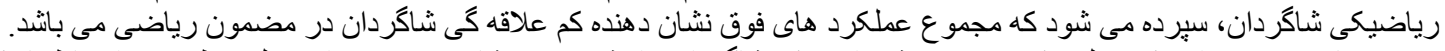

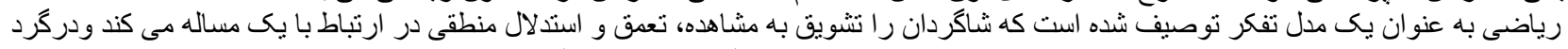

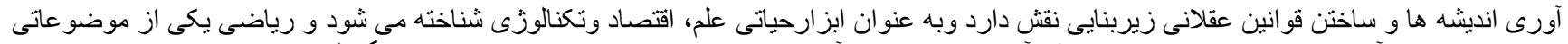

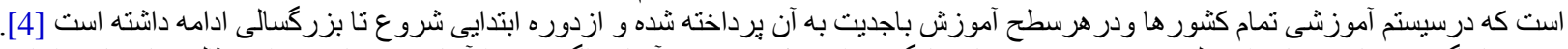

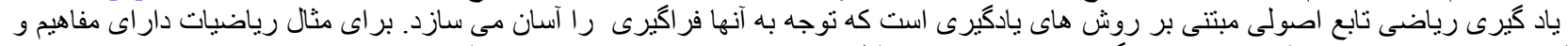

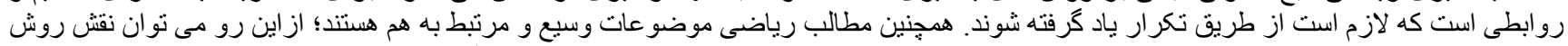

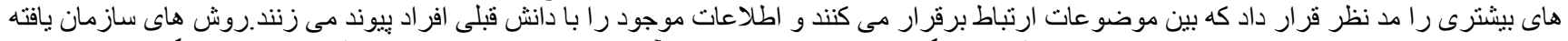

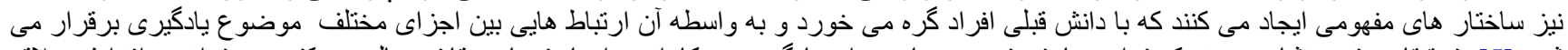

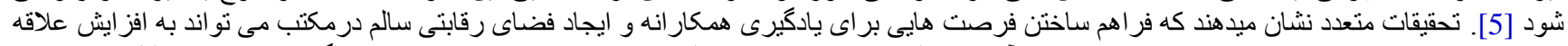

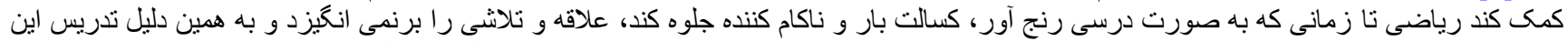

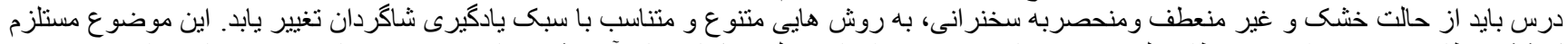

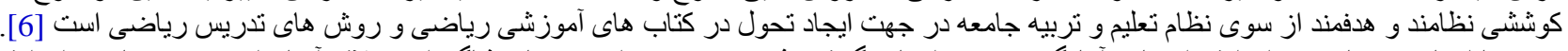

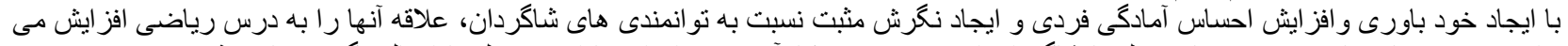

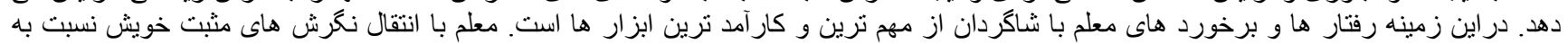

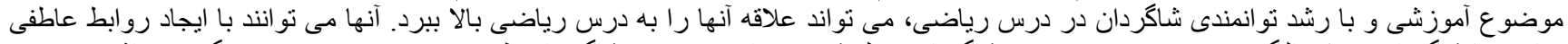

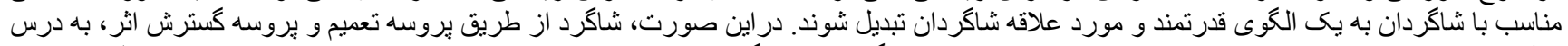

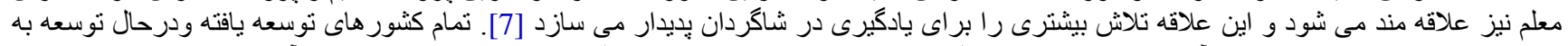

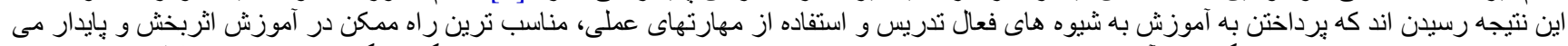

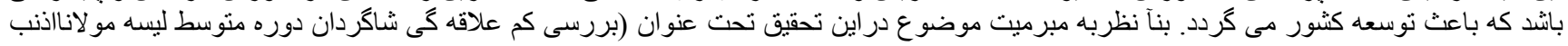


شبر غانى )، كم علاقه گَى شاكردان دوره منوسطه مكتب متذكره مورد بررسى قرار مى كيرد تا دريافت كردد كدام عوامل اساسى ازنظر شاكردان دوره

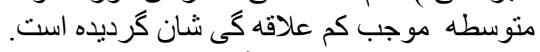

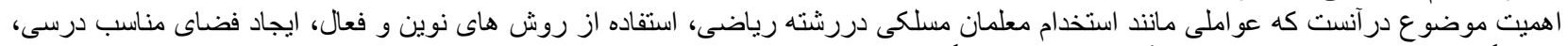

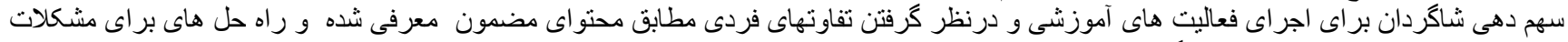

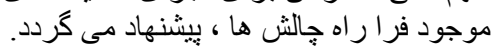

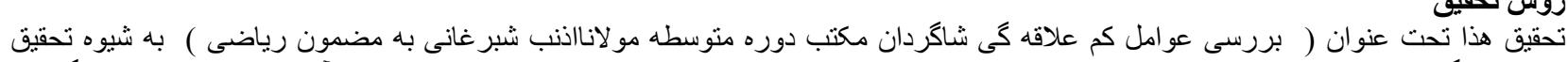

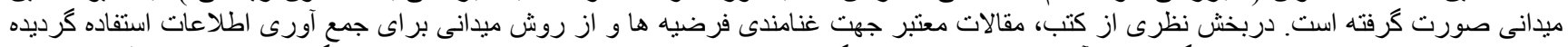

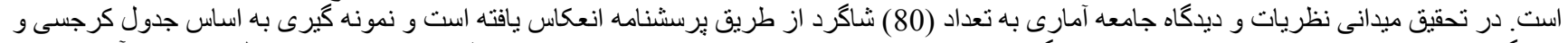

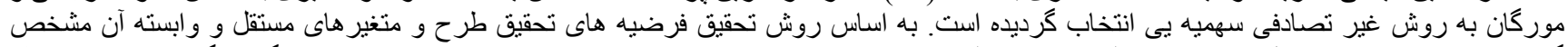

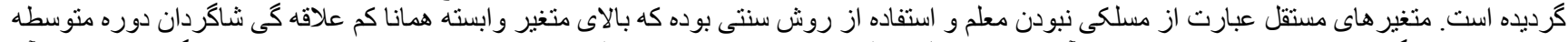

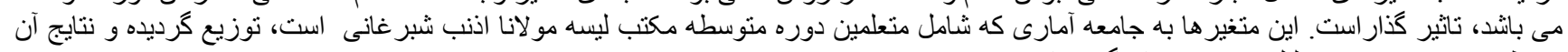

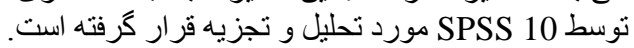

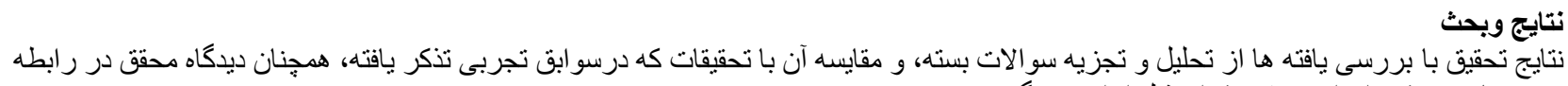

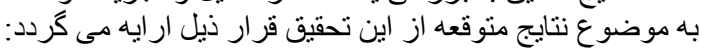

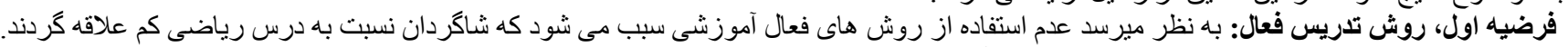

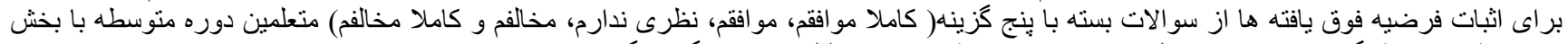

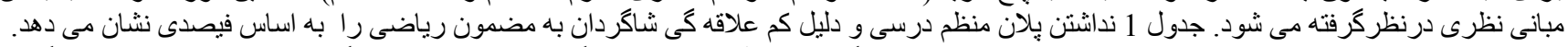

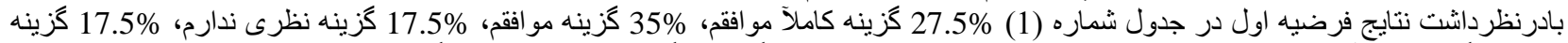

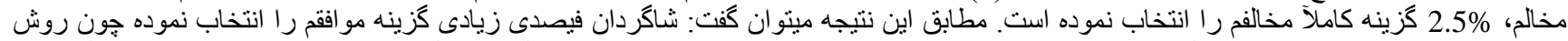

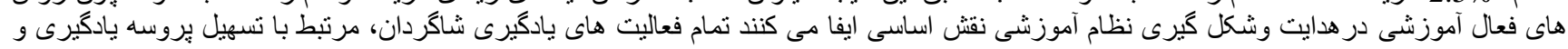

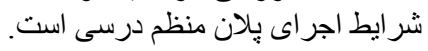

جدول 1: ميز ان عدم استفاده از روش هاى فعال آموزشى و نداشتن بلان منظم درسى كه سبب كم علاقه كى شاكردان كرديده است

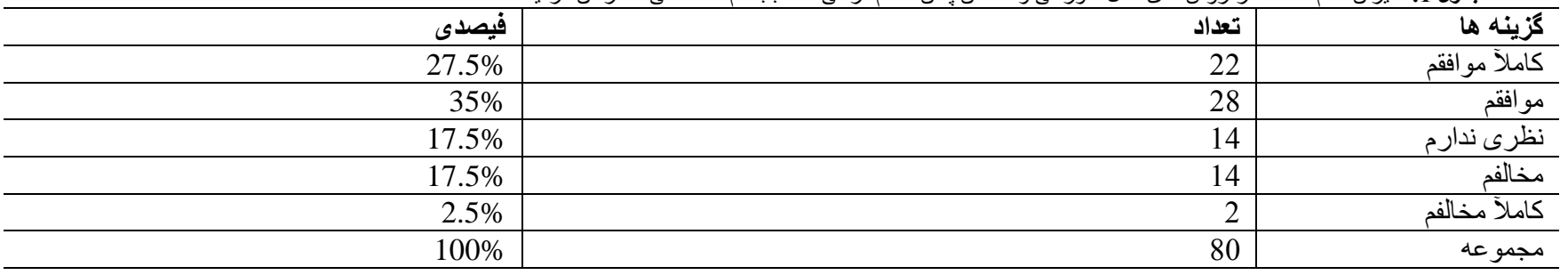

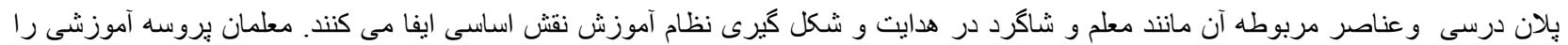

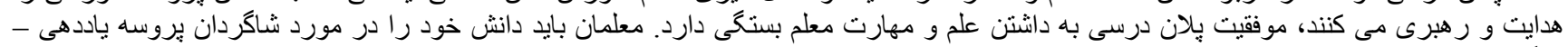

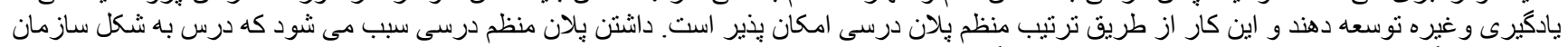

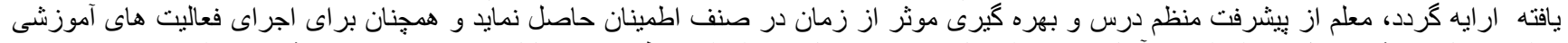

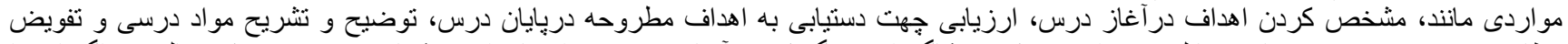

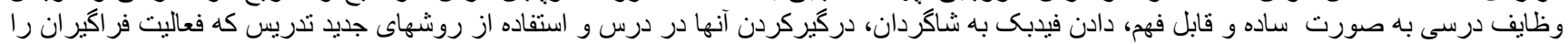

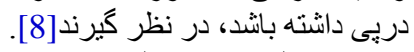

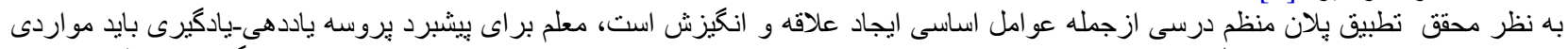

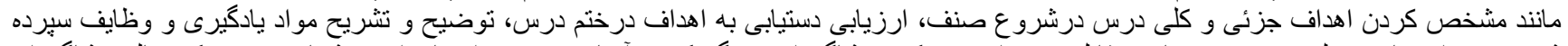

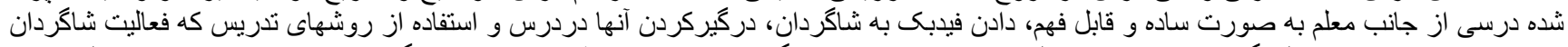

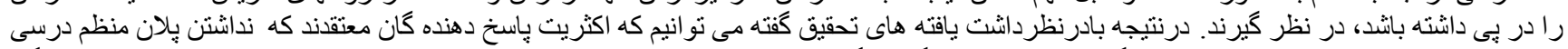

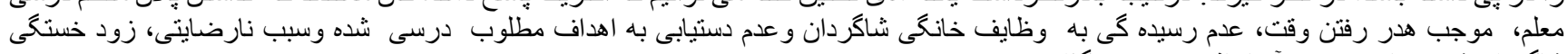

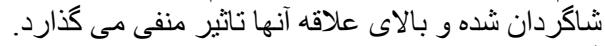

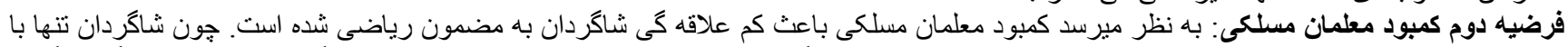

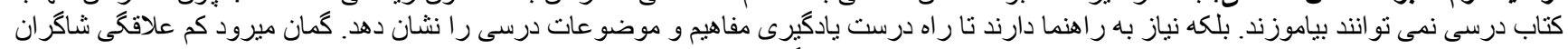

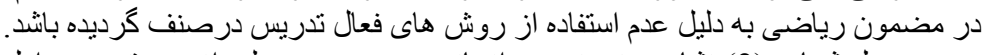

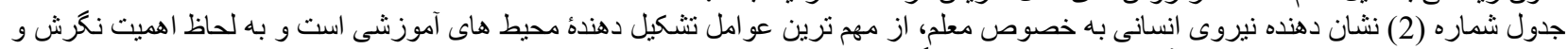

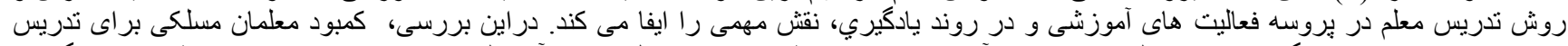

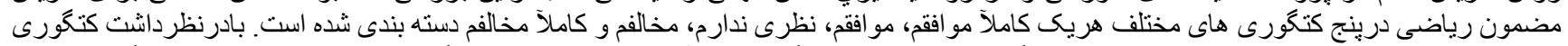

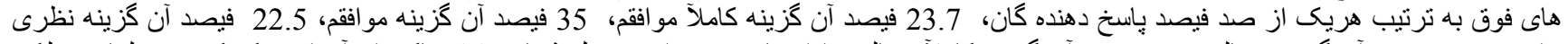

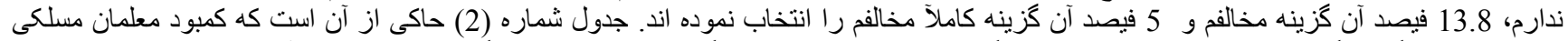

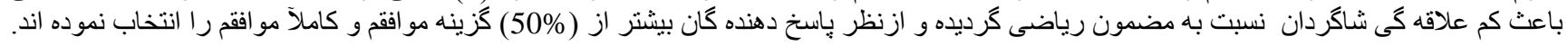


جدول 2: كمبود معلمان مسلكى باعث كم علاقه كَى شاكر دان نسبت به مضمون رياضى كرديده است.

\begin{tabular}{|c|c|c|c|}
\hline \multicolumn{2}{|c|}{ فيصدى } & \multirow{2}{*}{$\begin{array}{rr}\text { تعداد } & \\
& 19 \\
\end{array}$} & \multirow{2}{*}{ كزيلاًه مو افقم } \\
\hline 23.7 & $\%$ & & \\
\hline 35 & $\%$ & $\begin{array}{ll} & 28 \\
\end{array}$ & مو افقم \\
\hline 22.5 & $\%$ & 18 & نظرى ندارم \\
\hline 13.8 & $\%$ & 11 & مخالفم \\
\hline \multirow{2}{*}{\multicolumn{2}{|c|}{$\begin{array}{r}\% \\
100 \% \\
\end{array}$}} & 4 & كاملآ مخالفم \\
\hline & & 80 & مجمو عه \\
\hline
\end{tabular}

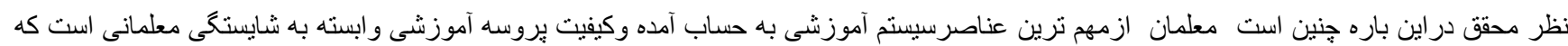

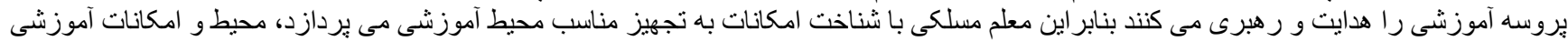

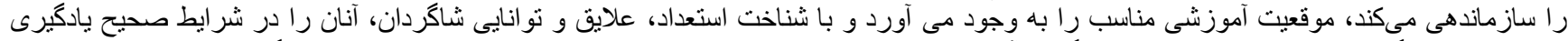

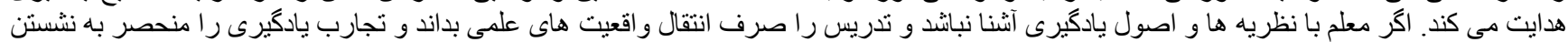

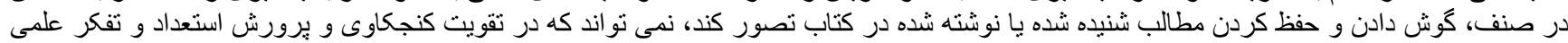

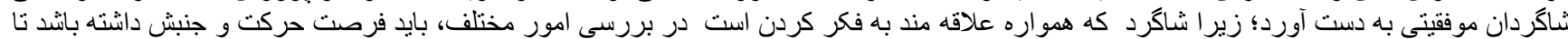

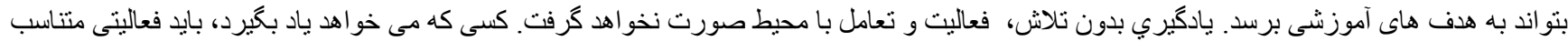

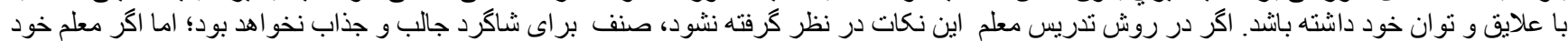

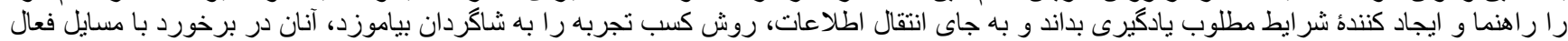

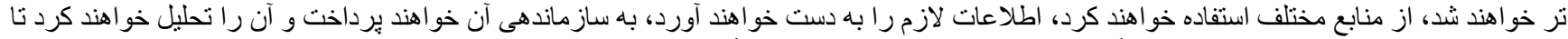

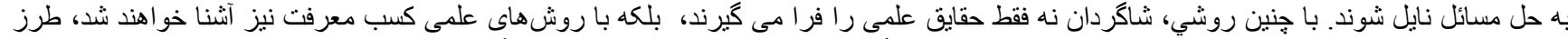

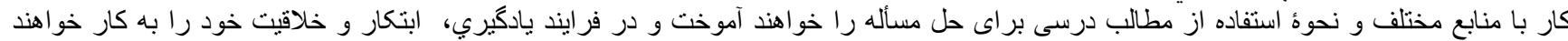

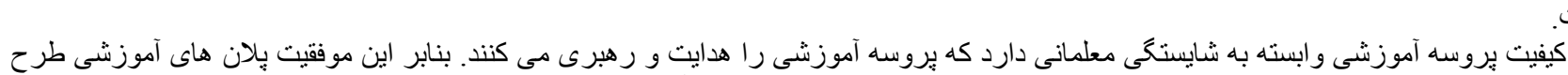

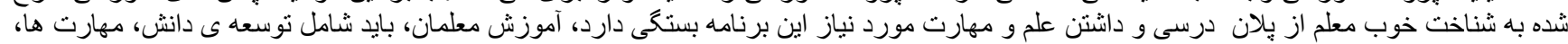

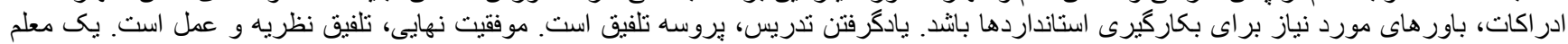

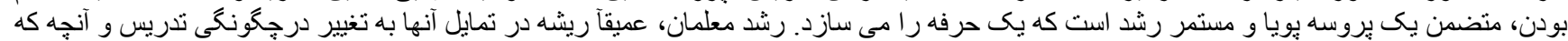

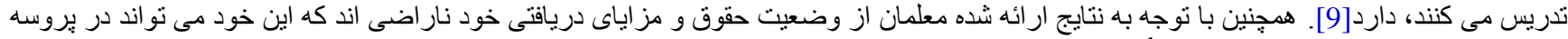

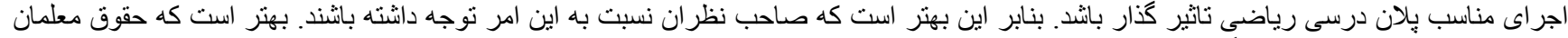

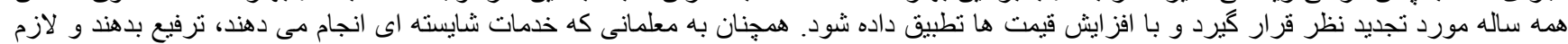

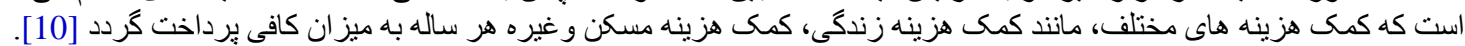

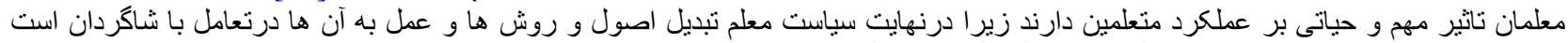

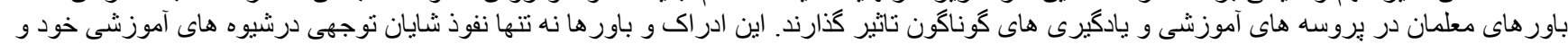

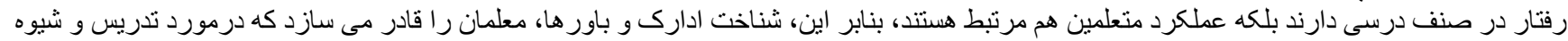

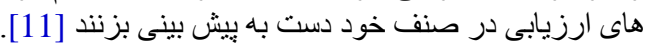

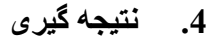

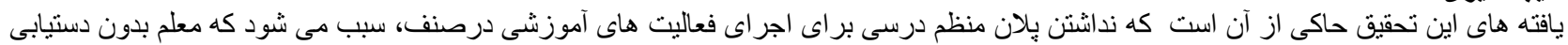

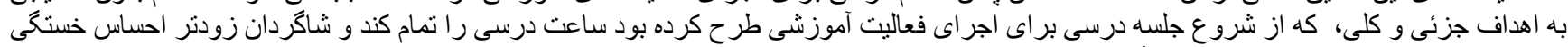

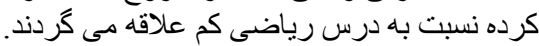

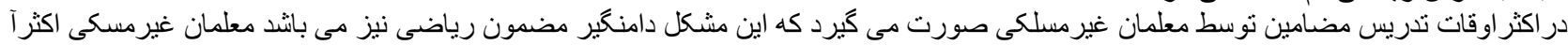

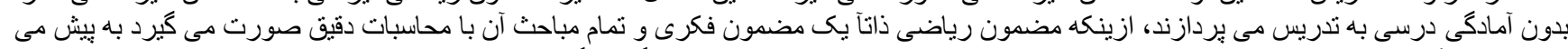

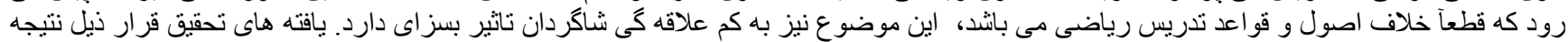

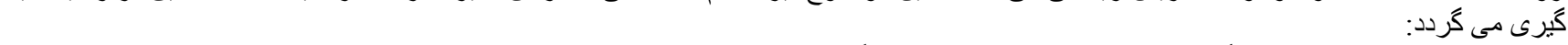

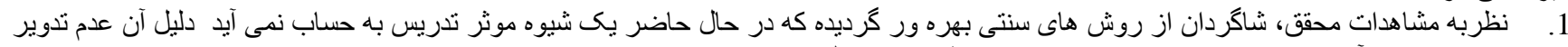

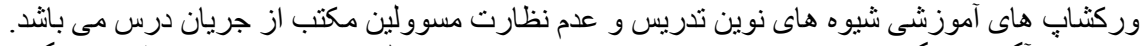

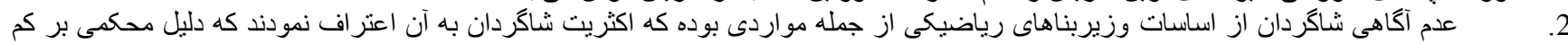

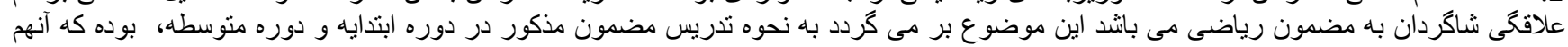

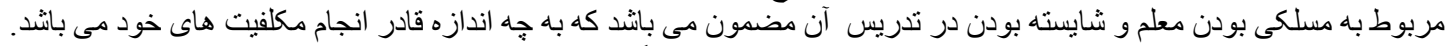

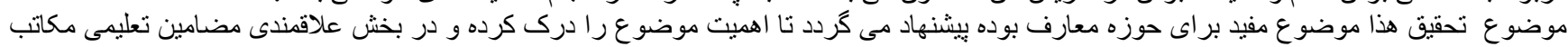
خصوصنا مضمون رياضى از نتايج آن استفاده نمايند.

\section{References}

[1] M. S. Qamrani, "The interest of the forgotten concept in education," Comprehensive Portal of Humanities, pp. 40-43, 2007.

[2] A. G. Rezaei and S. H. Pasha, "A model for creating interest and motivation in study and research, SID.ir," pp. 152-184, 2008.

[3] A. A. Hosseinkhanzadeh, S. Ebrahimi, F. Khodakarami, and A. Hasirchaman, "The effect of education through motor games on the learning math concepts and interest to mathematics in slow learner students," Journal of Psychological Science, vol. 18, pp.2019,797-806.

[4] S. A. Confectionery and S. Hesami, "Investigating the causal relationship between learning approaches and mathematical performance mediated by interest and behavioral preoccupation," Comprehensive Portal of Humanities, pp. 38-51, 1396.

[5] Etiquette, "Meta-analysis of relationships between learning strategies and mathematical problem solving performance," Comprehensive Humanities Portal, pp. 34-55, 2015.

[6] M. Vasel, "Predictors of mathematical anxiety and its relationship with mathematics failure," Comprehensive Humanities Portal, pp. 20-38, 2009. 
[7] A. Y. Rashid and R. K. Noghabi, "A study and comparison of the impact of how to design a classroom oral question for learning mathematics and students' interest in this course," Comprehensive Humanities Portal, pp. 126-153, 2011.

[8] M. J. L. Birmipoor, "Assessing the quality of teaching mathematics in the fourth grade of Isfahan primary school in order to provide solutions to improve students' performance in the thames international exam," Comprehensive Portal of Humanities, pp. 50-68, 2009.

[9] H. A. Rahimi, D. Qarizada, and A. H. Stanikzai, "Evaluation of teaching methods of chemistry concepts through laboratory work: A case study of chemistry department, faculty of natural sciences ,Shaheed Professor Rabbani Education University," International Journal of Innovative Research and Scientific Studies, vol. 1, pp. 52-62 2018.

[10] A. Karami and M. R. N. Afrooz, "Evaluation of math curriculum for students with mental retardation in Tehran," Comprehensive Portal of Humanities, pp. 5-21, 2013.

[11] Morvati, "The causal relationship between teacher self-efficacy and students' academic performance mediated by classroom management and teaching approaches," Comprehensive Humanities Portal, pp.2016 ,2-26 . 